EUROPEAN ORGANIZATION FOR NUCLEAR RESEARCH

CERN — AB DEPARTMENT

CERN-AB-2004-097 ADM

\title{
The CERN Antiproton Collider Programme: Accelerators and Accumulation Rings
}

\author{
H. Koziol and D. Möhl
}

\begin{abstract}
One of CERN's most daring and successful undertakings was the quest for the intermediate bosons, $\mathrm{W}$ and $\mathrm{Z}$. In this paper, we describe the accelerator part of the venture which relied on a number of innovations: an extension of the budding method of stochastic cooling by many orders of magnitude; the construction of the Antiproton Accumulator, depending on several novel accelerator methods and technologies; major modifications to the $26 \mathrm{GeV}$ PS Complex; and the radical conversion of the $300 \mathrm{GeV}$ SPS, which just had started up as an accelerator, to a proton-antiproton collider. The SPS Collider had to master the beam-beam effect far beyond limits reached ever before and had to function in a tight symbiosis with the huge detectors UA1 and UA2.
\end{abstract}

Submitted for publication in Physics Reports

Geneva, Switzerland

November 2004 


\section{Introduction}

An enlightening overview of the early ideas for the use of antiprotons in colliding-beam devices is due to Johnsen [1]. Two concepts had to mature and mate to lead to CERN's $\bar{p} \bar{p}$ Collider: particle-antiparticle colliders, and beam cooling. Proton-antiproton colliders were first discussed at CERN in 1962 [1], 7 years after antiproton discovery at the Bevatron in Berkeley. Considerations included the Intersecting Storage Rings (ISR), then in their early design stage, but with the expected densities of antiproton beams, luminosities were discouragingly low. Prospects changed in 1966, with Budker's [2] and O'Neill's [1, 2] ideas of electron cooling. In [2], Budker describes the application of his invention of electron cooling specifically for the production of antiproton beams dense enough to make proton-antiproton colliders viable. These need only a single ring, a concept already practiced at that time with electron-positron colliders. Rubbia, realizing the potential offered by the projected " $300 \mathrm{GeV}$ Machine" later built at CERN as the Super Proton Synchrotron (SPS), proposed, still in 1966, to use it as a proton-antiproton collider [1].

The next step occurred in 1968, when van der Meer invented stochastic cooling (published only in 1972 [3]). Both electron and stochastic cooling were experimentally proven in 1974, at the NAP-M storage ring in Novosibirsk [4], and at the CERN ISR [5], respectively. With these tools at hand, Rubbia et al. [6] revived the idea of converting the SPS into a protonantiproton collider, with the specific aim of producing the long-awaited $W$ and $Z$ bosons. At that stage, his scheme was still based on electron cooling.

Rubbia's enthusiastic promotion led to the Initial Cooling Experiment (ICE) [7], a $2 \mathrm{GeV}$ storage ring, quickly built during 1977, using the magnets of the g-2 experiment. Early in 1978, stochastic cooling proved so successful (Fig. 1) that the collider scheme was then entirely based on it. It should be pointed out that already in 1976, Strolin et al. [8] had proposed an accumulator ring using stochastic cooling, to provide dense antiproton beams for the ISR.

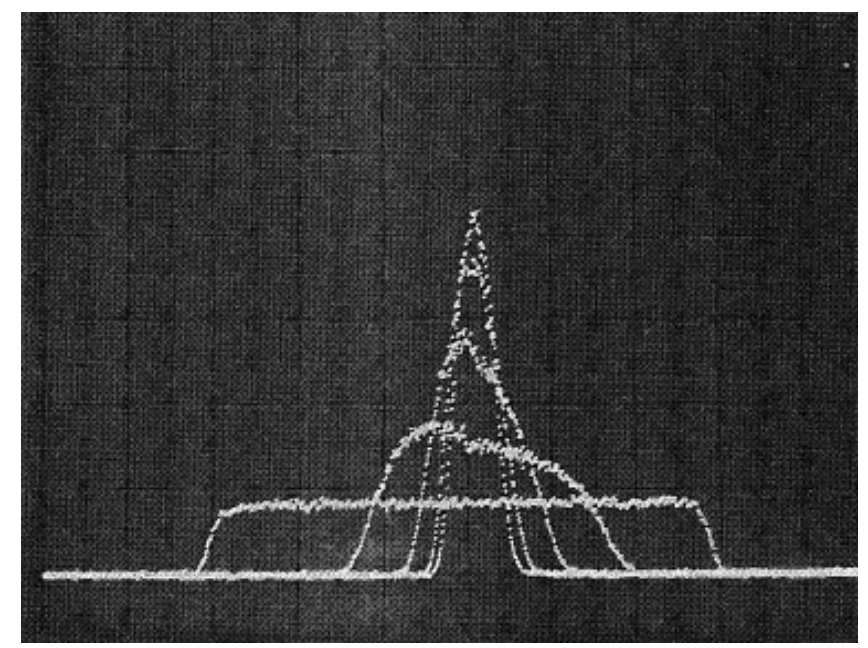

Fig. 1 - Demonstration of stochastic momentum cooling in ICE. The momentum distribution of $5 \times 10^{7}$ circulating protons was first spread out by applying noise around a harmonic of the revolution frequency on an RF-cavity (1st Schottky scan, rectangular shape). Then cooling was turned on. Scans at $1 \mathrm{~min}$ intervals show the concentration of the protons into a narrow momentum bite. The signal height is proportional to the square root of particle density, and the width of the trace is proportional to $\Delta p / p$. 
Stochastic cooling in ICE, apart from increasing the density of the beam, extended its life time, given by Coulomb scattering on the residual gas, from hours to days. This permitted another striking proof [9]: CPT stipulates identical decay-lifetimes for antiprotons and protons, practically infinite compared to the days of accumulation time. However, the experimental lower limit for antiprotons was only $120 \mu \mathrm{s}$ (derived from bubble chamber tracks). Despite faith in theory, it was a nightmare to launch a multi-million project in such a situation. To remove doubts, a beam of 240 antiprotons was therefore stored and stochastically cooled in ICE. Four days later, 80 were still circulating. This established a new lower limit for the decay-lifetime of $32 \mathrm{~h}$ at rest, an improvement by 9 orders of magnitude and a relief for those who needed more than just faith in CPT.

The early result from ICE led to a rapid decision to go ahead. With stochastic cooling, antiprotons could be cooled and accumulated directly at $3.5 \mathrm{GeV} / \mathrm{c}[\underline{10}, \underline{11}]$, where the yield from $26 \mathrm{GeV}$ (PS) protons is highest. The overall scheme (see chapter 2 and Fig. 2), involved the construction of the Antiproton Accumulator (AA) and major modifications to the PS and SPS.

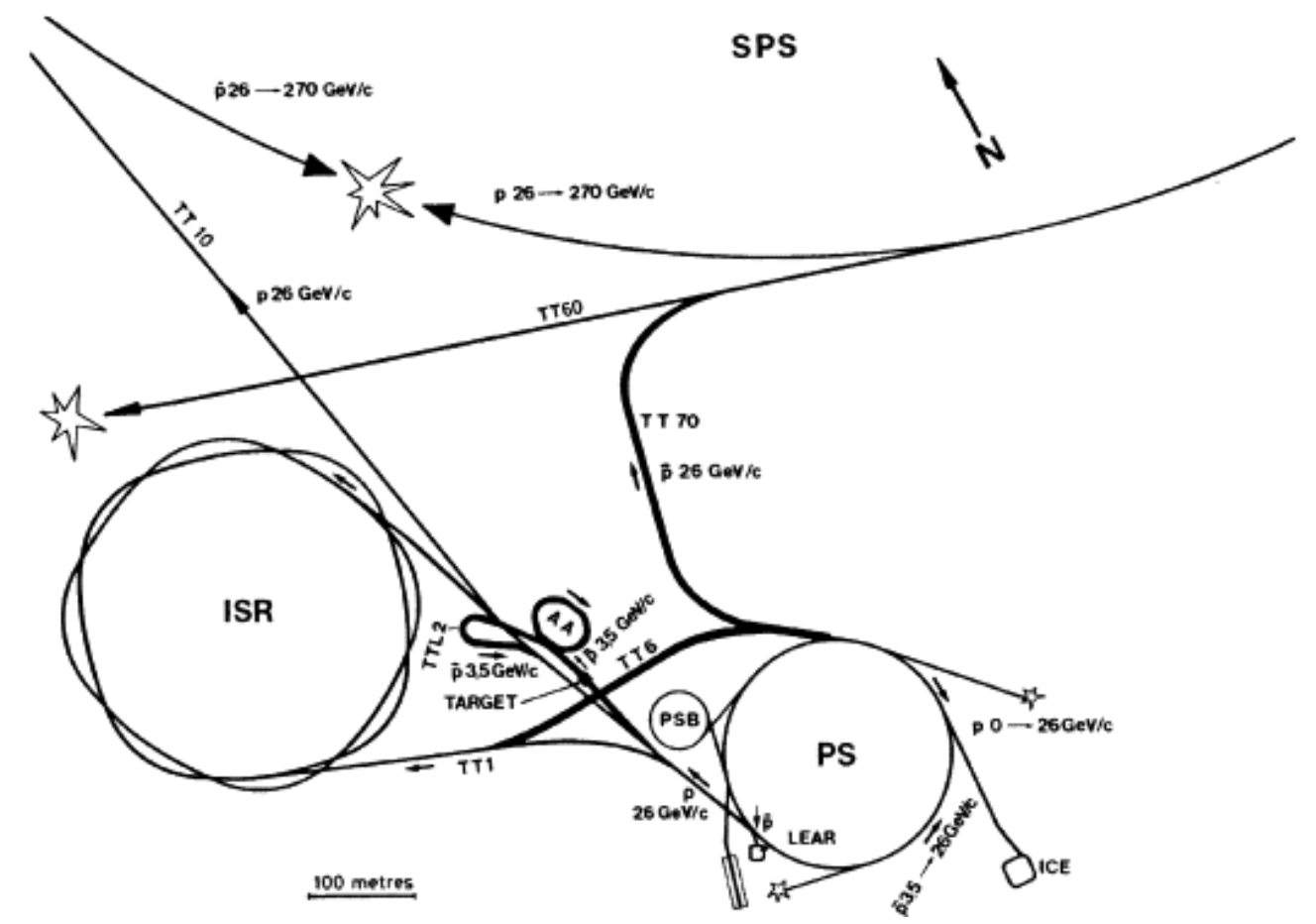

Fig. 2 - Layout of CERN accelerators in 1981. Antiproton paths appear in heavier lines. LEAR, still under construction in 1981, is also shown.

Construction of the AA began in 1979. In April 1981, the first proton-antiproton collisions occurred in the ISR, at $2 \times 26 \mathrm{GeV}$. The SPS followed on the heels, with collisions at $2 \times 273$ GeV on 10 July 1981 . The first $W$ data were taken in 1982 and the discovery of the $W$ and $Z$ was announced in 1983. In order to satisfy the ever-increasing appetite of antiproton users, the Antiproton Collector (AC) [12] was built around the AA in 1986. From 1987 on, it boosted the accumulation rate, eventually by an order of magnitude. After a last collider run in 1991, the SPS returned to an accelerator-only life.

The Low-Energy Antiproton Ring (LEAR) [13] continued to take beam from the AC/AA until end 1996. In 1997, the AA was dismantled and the AC converted into the Antiproton Decelerator (AD) [13], to provide low-energy antiprotons in a simpler way. 


\section{Outline of the scheme}

The $50 \mathrm{MeV}$ Linac, the $800 \mathrm{MeV}$ Booster and the $26 \mathrm{GeV}$ PS (Fig. 2) were pushed hard to deliver an intense proton beam on the production target. The burst of antiprotons emerging around $3.5 \mathrm{GeV} / c$ was captured in the AA (or, after 1987, in the AC).

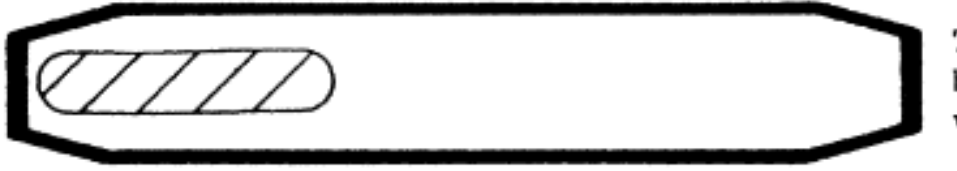

The first pulse of $7 * 10^{6} \bar{p}$ has been injected into the vacuum chamber.

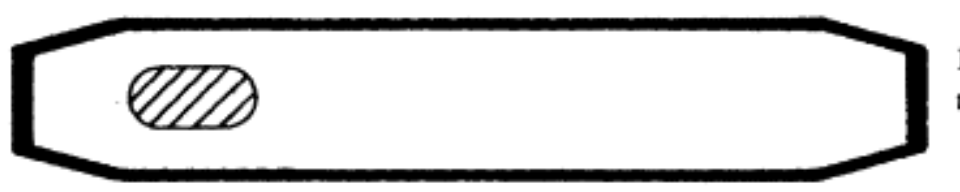

Precooling has reduced the momentum spread.

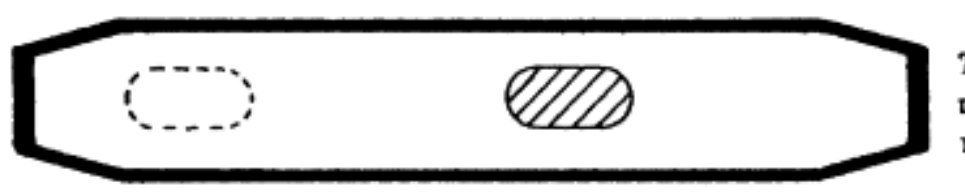

The first pulse has been moved to the stack-tail region.

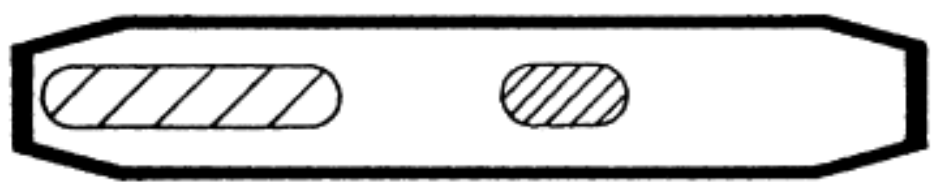

The second pulse is injected, $2.4 \mathrm{~s}$ later.

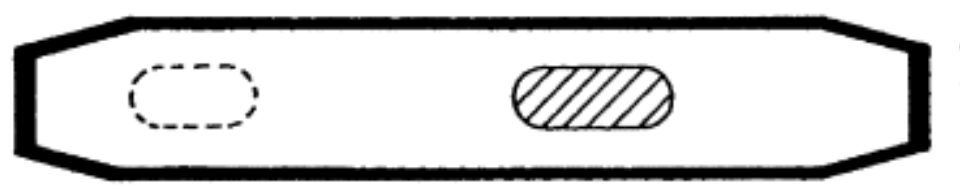

The second pulse, after having been precooled, is also stacked.

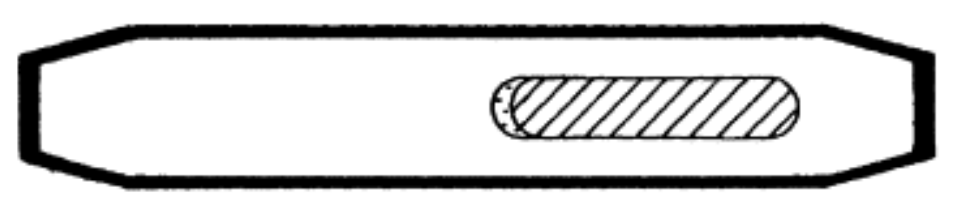

After 15 pulses, the stack contains $10^{8} \overline{\mathrm{p}}$.

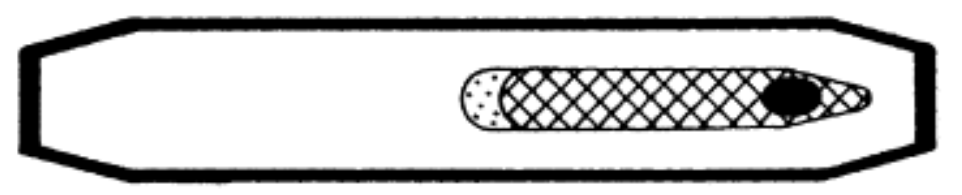

After $1 \mathrm{~h}$, a dense core has formed inside the stack.

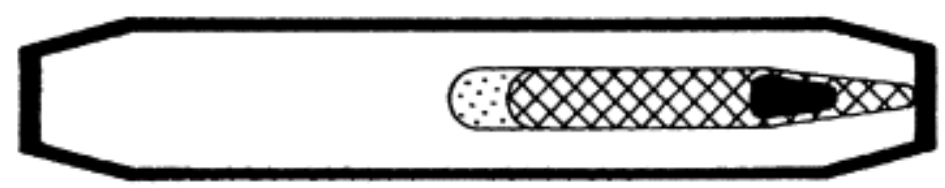

After 1 day, the core contains enough antiprotons for transfer to the SPS.

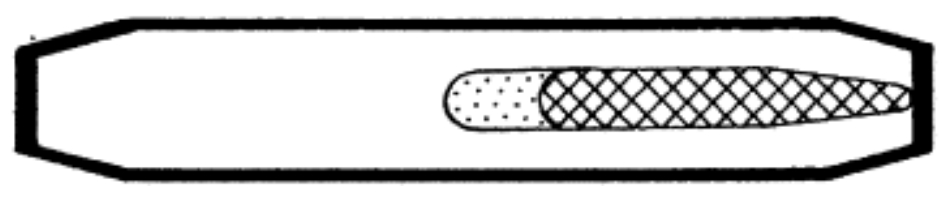

The remaining antiprotons are used to begin the next day of accumulation.

Fig. 3 - Schematic sequence illustrating cooling and accumulation in the original AA, before the advent of the AC. (From [11]) 
In the original AA, a fresh burst of antiprotons remained on the injection orbit for at least $2 \mathrm{~s}$ for stochastic precooling of the momentum spread. The radiofrequency system then trapped and moved them to the stacking region where stack-tail cooling took over. The injection region was now free for the next burst of antiprotons, arriving 2.4 or $4.8 \mathrm{~s}$ later. This sequence was repeated during the whole accumulation period (Fig. 3).

In the AC, a powerful "bunch rotation" RF system $(1.5 \mathrm{MV}, 9.5 \mathrm{MHz})$ turned the incoming 5 antiproton bunches into a nearly continuous beam of lesser momentum spread. Stochastic cooling in all three planes then reduced the emittances by large factors. Another RF system $(3.5 \mathrm{kV}, 1.6 \mathrm{MHz})$ re-bunched the antiprotons, for ejection and transfer into a matched bucket on the AA injection orbit.

Both in the original and the modified AA the antiproton stack was subjected continuously to up to six different stochastic cooling systems. Over a day, a stack with a dense core of several $10^{11}$ antiprotons was accumulated.

An antiproton bunch was picked from the stack by creating an "unstacking bucket" in the core region and moved to the ejection orbit. From there it was sent through a "loop" (TTL2, Fig. 2) to the PS. For use in the Collider it was accelerated to $26 \mathrm{GeV} / c$ and sent to the SPS as described in Section 3.1.3 below. Prior to a transfer, careful checks were performed, concluded by the dispatch of a small "pilot bunch" of $\sim 10^{9} \overline{\mathrm{p}}$ all the way from the AA to the SPS. This assured that the big shot, containing a day's harvest, would safely find its way.

Transfers of antiprotons to LEAR were made in a different way, described in [13].

\section{Antiproton production and accumulation}

\subsection{The rôle of the PS}

\subsubsection{High-intensity primary proton beam}

To produce a large number of antiprotons demands a proton beam of an intensity as high as the target will stand. The transverse emittances have to be small, to permit focusing to a small size over the whole length of the target. Finally, the proton burst length had to correspond to the AA circumference, $1 / 4$ that of the PS.

This meant that the PS beam, normally 20 bunches around the whole circumference, had to be crowded into 5 bunches. The fact that the $800 \mathrm{MeV}$ PS Booster consists of 4 superposed rings, each $1 / 4$ of the PS circumference, was helpful. The beams from 2 Booster rings (5 bunches each) were ejected simultaneously, combined in the vertical plane, and injected into the PS. This was repeated with the other 2 rings, timed such that 2 sets of 5 bunches circulated on opposite sides of the PS. After acceleration to $26 \mathrm{GeV} / c$, on the flat top, one set was slightly accelerated and advanced towards the other one. When the 2 sets had fallen into step, they were ejected towards the target. Later, for the $\mathrm{AC}$, different techniques of bunch merging were used, but the goal, to compress the beam into 5 bunches distributed over $1 / 4$ of the PS circumference, remained the same.

Nominally, that process was repeated every $2.4 \mathrm{~s}$, but usually the interval was extended to 3.6 or $4.8 \mathrm{~s}$, in order to give more time to cooling in the AA, and to allow the PS to serve other users. The intensity was raised from a prudent $10^{13}$ to finally $1.3 \times 10^{13}$ protons per pulse. 


\subsubsection{Post-acceleration of antiprotons}

The momentum of $3.5 \mathrm{GeV} / c$ was too low for direct transfer to the SPS. Therefore, the antiprotons, extracted in single bunches from the AA, were sent to the PS via the newly built "loop" (TTL2, Fig. 2), for acceleration to $26 \mathrm{GeV} / c$. Subsequent "bunch rotation" reduced their length to about 4 ns before transfer to the SPS through the new line TT70 (Fig. 2). This process was repeated every $2.4 \mathrm{~s}$, until 3 (later 6) antiproton bunches were circulating on the SPS injection orbit, equidistantly spaced. For safety, the transfer of the same number of "cheap" proton bunches preceded that of the "precious" antiprotons.

\subsubsection{Proton test beams for $A A$ and $A C$}

Initial setting-up of the AA (later also of the AC) was done with protons. For this purpose, the PS produced single bunches at $3.5 \mathrm{GeV} / c$, which first followed the path of the $26 \mathrm{GeV} / c$ protons towards the target location and then, with the target removed, through the antiproton injection line to the AA (later the AC), which for that purpose had all magnets in "opposite polarity".

Precision setting-up of the AA and AC magnets had to be done in "normal polarity", and the $3.5 \mathrm{GeV} / c$ proton test beam from the PS came through the loop (TTL2). This was done also during routine operation, preceding antiproton transfers from the AA to the PS, in order to verify the correct settings of the AA ejection and of the channels.

All the exercises described above demanded precise tuning of the machines to each other, in terms of magnetic field, orbit position and revolution frequency. Timing logics was intricate, timing precision had to be high, and all the settings had to be flipped from one operational mode to the other, within $1.2 \mathrm{~s}$.

\subsection{Antiproton production}

Antiprotons are produced by a high-energy proton beam hitting a target. The yield is highest at a momentum, $\mathrm{p}_{\overline{\mathrm{p}}}$, corresponding to production at rest in the centre-of-mass system of the incoming proton and a stationary target nucleon [14]. For $26 \mathrm{GeV} / \mathrm{c}$ protons there is a flat maximum at $\mathrm{p}_{\overline{\mathrm{p}}} \approx 3.5 \mathrm{GeV} / \mathrm{c}$. To calculate the conversion efficiency one uses the "differential yield" $[\underline{14}, \underline{15}]$, i.e. the differential cross section for antiproton production divided by the cross section for inelastic scattering

$$
\frac{\mathrm{d}^{2} N_{\overline{\mathrm{p}}}}{\mathrm{d} \Omega \mathrm{d} p}=\frac{1}{\sigma_{\text {inel }}} \frac{\mathrm{d}^{2} \sigma_{\overline{\mathrm{p}}}}{\mathrm{d} \Omega \mathrm{d} p}\left(\operatorname{sterad}^{-1}(\mathrm{GeV} / \mathrm{c})^{-1}\right) .
$$

It represents the number of antiprotons produced per interacting proton, per unit of solid angle $\mathrm{d} \Omega$, and per momentum bite $\mathrm{d} p$ (around the maximum at $\mathrm{p}_{\overline{\mathrm{p}}}$ ). Differential yields have been calculated and measured for various materials and momenta $[\underline{14}, \underline{15}]$ and were found to rise steeply with proton momentum, before beginning to level off at about $100 \mathrm{GeV} / c$ (e.g. for tungsten, one has $0.013 \operatorname{sterad}^{-1}(\mathrm{GeV} / c)^{-1}$ at $26 \mathrm{GeV} / c(\mathrm{CERN})$, and 0.25 at $120 \mathrm{GeV} / c$ (FNAL production beam [16]).

Target material and geometry are chosen to maximize proton interactions, while minimizing antiproton re-absorption. Also, beam optics before and after the target is vitally important. The result was a rod-shaped target (Fig. 4), made of a heavy material, with a diameter of 
about $3 \mathrm{~mm}$ and a length, $L_{\mathrm{t}}$, between 50 and $120 \mathrm{~mm}$, of the same order as the inelastic collision length, $\left(L_{\mathrm{t}} \approx L_{\text {inel }}\right)$. This gives a "target efficiency" $N_{\text {interact }} / N_{\text {incident }}=1-\exp \left(-L_{\mathrm{t}} / L_{\text {inel }}\right)$ of about 0.6 , provided that the primary proton beam is focused to a small size over the entire length of the target.

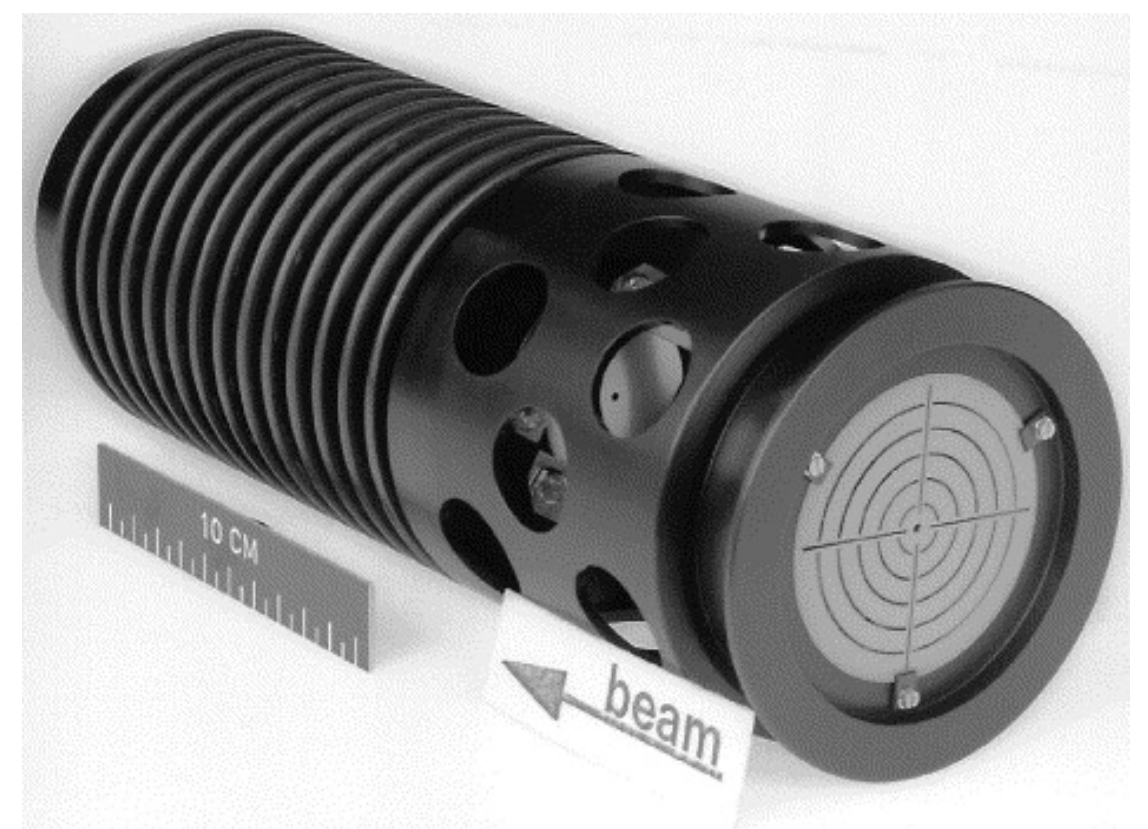

Fig. 4 - An antiproton production target for the AA (1980). The tungsten rod, $110 \mathrm{~mm}$ long and $3 \mathrm{~mm}$ in diameter, is embedded in graphite, pressed into an aluminium container with cooling fins for forced air cooling. Correct position and size of the $26 \mathrm{GeV}$ proton beam from the PS was monitored on a scintillator screen, with circles every $5 \mathrm{~mm}$.

The channel following the target has to match the "antiproton line source" (target) to the acceptance of the ring (AA, later $\mathrm{AC})$. This is obtained by making the horizontal and vertical beta functions at the target about equal to its length $\left(\beta_{\mathrm{h}} \approx \beta_{\mathrm{v}} \approx L_{\mathrm{t}}\right)$. Ideally, the captured solid angles are then determined by the transverse acceptances of the ring $\left(E_{\mathrm{h}}, E_{\mathrm{v}}\right.$ in units of $\pi \mathrm{m} \mathrm{rad})$ through $\Delta \Omega \approx \pi \sqrt{E_{\mathrm{h}} E_{\mathrm{v}} / L_{\mathrm{t}}^{2}}$, while the momentum-bite $\Delta p$ is the momentum acceptance of the ring.

The yield per incident proton $\mathrm{Y}=N_{\overline{\mathrm{p}}} / N_{\mathrm{p}}$, obtained by multiplying the above expressions for target efficiency, differential yield (at its maximum), momentum bite, and angular acceptance, is rather an upper limit: the matching of the primary beam to the target is not perfect, neither is it for the secondary beam to the ring; the differential yield diminishes at large angles and large deviations from optimum momentum; there is re-absorption inside the target.

For the original AA (as in 1984) with a $120 \mathrm{~mm}$ long copper target and the acceptances listed in chapter 3.3, calculation, assuming maximum yield and perfect matching, gives $Y \approx 10^{-6}$. Measurement gave $0.6 \times 10^{-6}$, with a magnetic horn (Fig. 5) as collector lens after the target. In the $\mathrm{AC}$, with its larger acceptances and a $60 \mathrm{~mm}$ long iridium target, the calculated value is $Y \approx 12 \times 10^{-6}$, whereas the best obtained was $6 \times 10^{-6}$ with a lithium lens and $5 \times 10^{-6}$ with a magnetic horn. These examples illustrate the degree to which "target matching" approached perfection. 

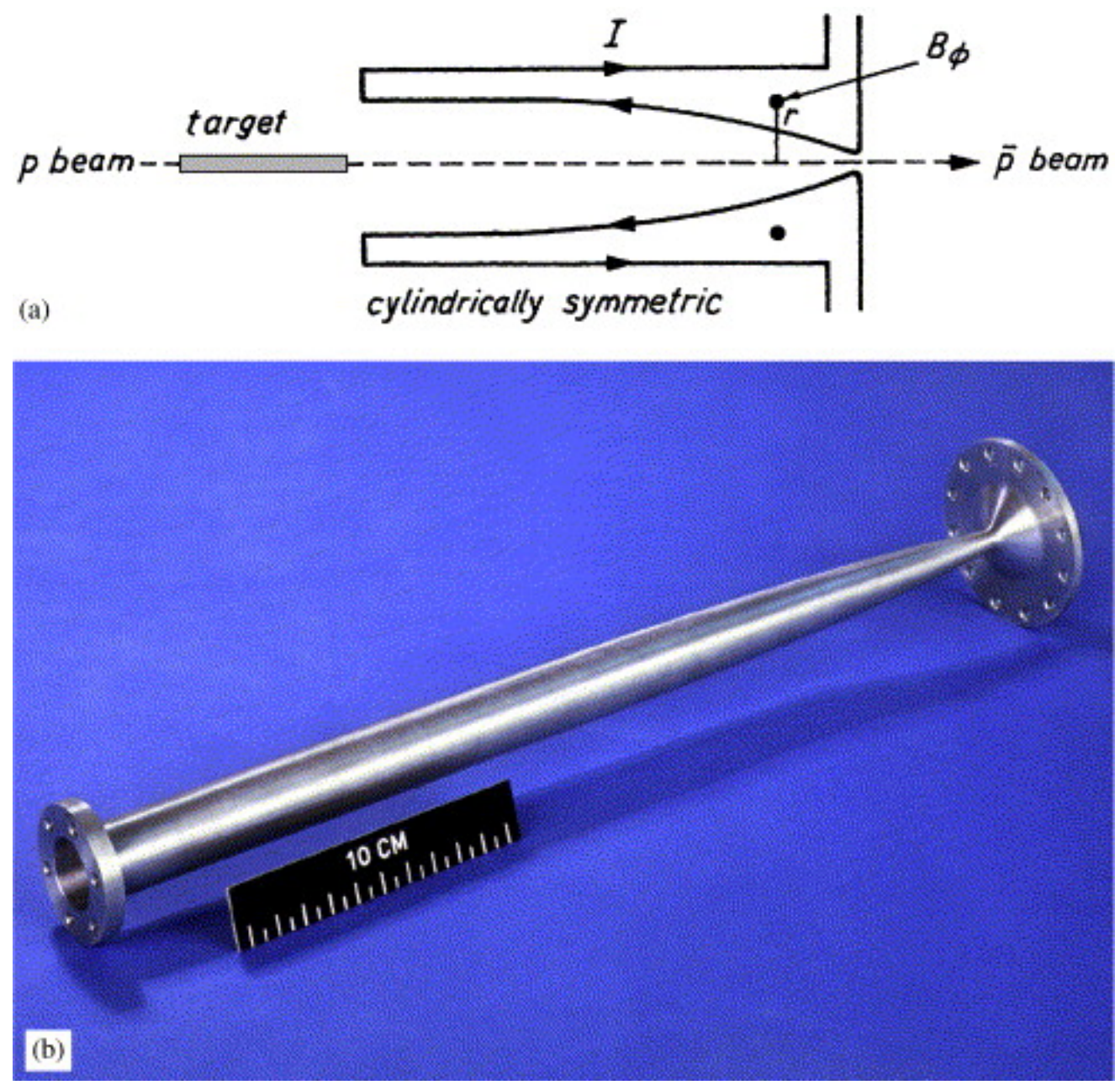

Fig. 5 - The magnetic horn of the AA collected antiprotons emitted at large angles from the target: (a) (from [11]) the flow of electrical current in a magnetic horn and (b) the inner conductor. The horn was pulsed at $400 \mathrm{kA}$ for $15 \mu \mathrm{s}$ (half-sine).

The small-emittance primary beam was matched to the target with quadrupoles and, for a certain period, with an additional lithium-lens. For capturing the antiprotons from the target one used magnetic horns [17, 18], (Fig. 5), lithium lenses [19], and an experimental plasma lens [20]. Magnetic horns proved more robust and easier to replace in the highly radioactive target area. Lithium lenses gave somewhat better yield. Early operation of the AA was with a horn, then lithium lenses were used for several years, and again horns for the late AC period and for the $\mathrm{AD}$.

A magnetic horn $[\underline{17}, \underline{18}]$ is a "current-sheet lens". Figure 5 shows its principle (a) and a picture (b) of its inner wall ("horn membrane"). A current flowing through the inner wall and returning via the outer one creates an azimuthal magnetic field in the space between them, but no field inside the horn. Antiprotons produced at large angles traverse the inner wall and are bent towards the axis. The horn membrane had to be thin to avoid scattering and absorption of the penetrating particles. It was made from aluminum, about $1 \mathrm{~mm}$ thick. The pulse currents were several $100 \mathrm{kA}$.

Lithium lenses [19] are rods of lithium, chosen for its low interaction with protons and antiprotons, surrounded by a pulse transformer, which induces a current along the axis. The azimuthal magnetic field inside and outside the rod focuses the particles. Rods of $34 \mathrm{~mm}$ 
diameter were used during SPS Collider operation, a $20 \mathrm{~mm}$ version for operation with LEAR alone. The pulse currents were also several $100 \mathrm{kA}$.

\subsection{The $\mathrm{AA}$ and $\mathrm{AC}$ rings}

The AA $[\underline{10}, \underline{11}, \underline{21}]$, (Fig. 6) had a circumference of $157 \mathrm{~m}$. Its magnetic field was constant, for a beam momentum of $3.5 \mathrm{GeV} / c$ on central orbit. It was built in a new hall and later buried under concrete shielding blocks. The optical properties were quite particular, dictated by the large acceptances required for antiproton collection and the needs of stochastic cooling and stacking. This leads to an impressively wide horizontal aperture, as much as $0.7 \mathrm{~m}$ in the regions of large dispersion where the orbit position depends strongest on momentum, providing spatial separation between the stack and the newly injected beam.

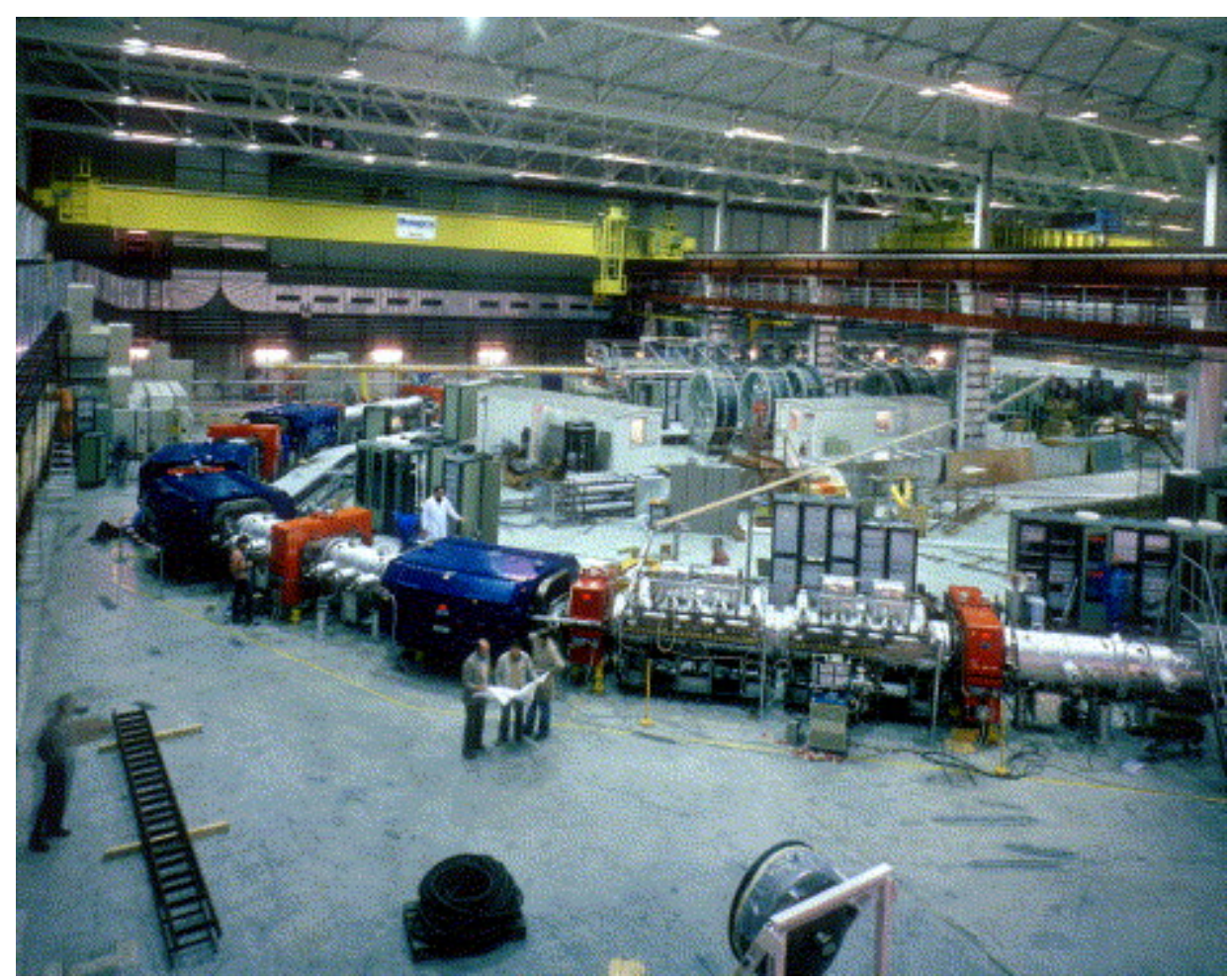

Fig. 6 - The AA in its hall, before it disappeared from view under heavy concrete shielding.

Quadrupoles and bending magnets (weighing 11 and $75 \mathrm{t}$, respectively) were large in those regions, but of conventional size in the less dispersive regions. Sections with vanishingly small dispersion were required for the location of kickers for momentum cooling of the stack, to avoid "heating" of the horizontal emittance. The injection kicker and the precooling devices had shutters for electromagnetic separation from the stack. Ultra-high vacuum $\left(10^{-11}\right.$ Torr) assured long storage times and, together with an elaborate clearing system, helped to reduce beam instability caused by ions trapped in the antiproton beam.

The AC [12, 22], (Fig. 7) with a circumference of $187 \mathrm{~m}$, was built in 1986/87 around the AA. Its primary task was to capture an order of magnitude more antiprotons than the AA, for which it had much larger acceptances (Table 1), both transverse and in momentum. 


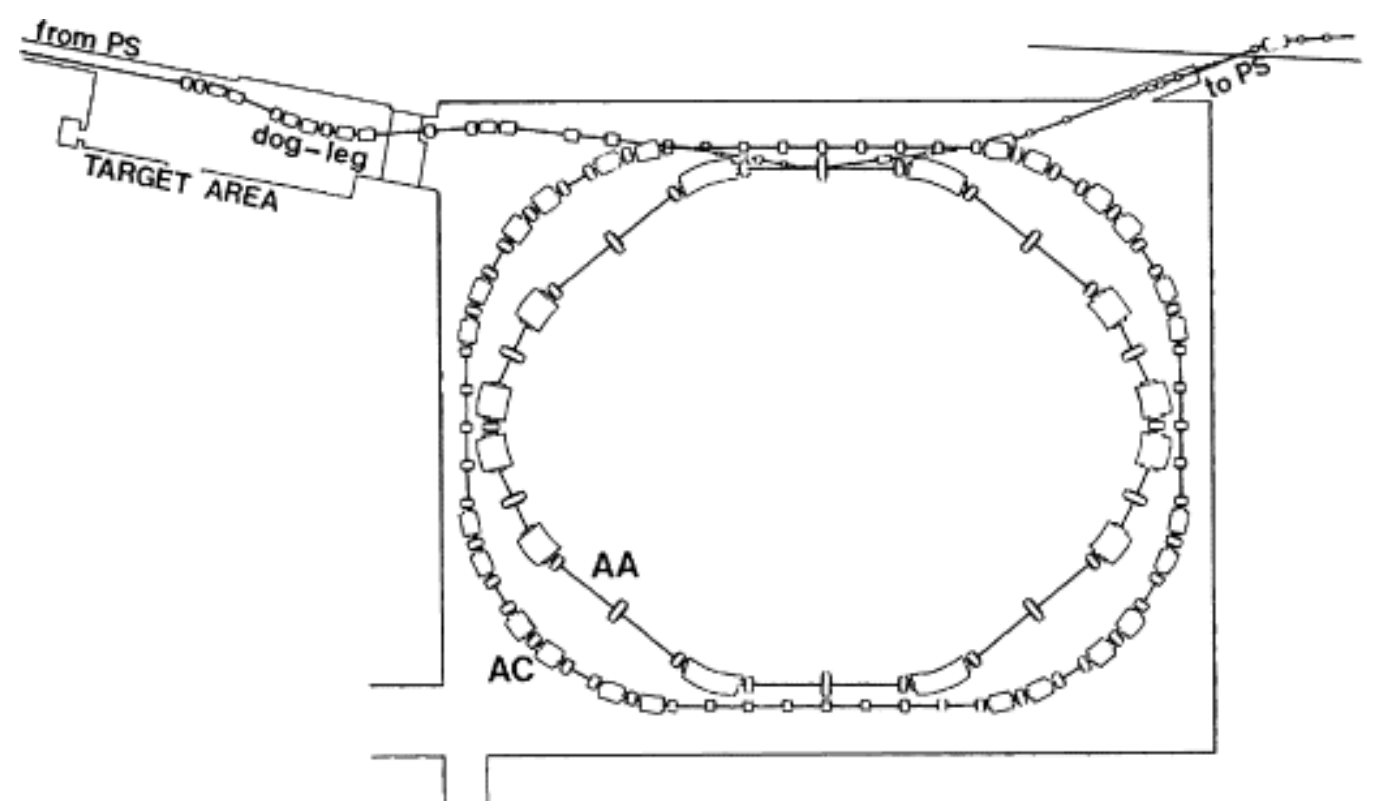

Fig. 7 - In 1986/87, the AC was built around the AA and a "dog-leg" incorporated in the injection line to diminish the flux of electrons and $\pi^{-}$reaching the hall. (From [11].)

Table 1 - Acceptances of AA and AC

\begin{tabular}{|l|c|c|c|}
\hline \multirow{2}{*}{ Ring } & \multicolumn{3}{|c|}{ Acceptances attained simultaneously } \\
\hline & Horizontal $\boldsymbol{E}_{\mathbf{h}}(\boldsymbol{\pi} \mathbf{~ m m}$ mrad $)$ & Vertical $\boldsymbol{E}_{\mathbf{v}}(\boldsymbol{\pi} \mathbf{~ m m}$ mrad $)$ & Momentum $\Delta \boldsymbol{p} / \boldsymbol{p}(\%)$ \\
\hline $\mathrm{AA}$ & 85 & 85 & \pm 0.75 \\
\hline $\mathrm{AC}$ & 200 & 200 & \pm 3.00 \\
\hline
\end{tabular}

A very strong focusing lattice was needed to provide such a large acceptance within an aperture not exceeding $0.35 \mathrm{~m}$. The AC also had regions with strong dispersion and others with zero dispersion, required by stochastic cooling, but in a less extreme way than the AA.

Two large and powerful cavities (each $2 \mathrm{~m}$ long and $2.5 \mathrm{~m}$ in diameter; together $1.5 \mathrm{MV}$ at 9.5 MHz) were installed in a dispersion-free straight section. After injection they "rotated" the bunches in longitudinal phase space, extending their length and reducing the momentum spread.

In the $\mathrm{AC}$, a vacuum of a $10^{-8}$ Torr was sufficient to avoid blow-up due to scattering as well as ion-trapping by the low-intensity antiproton beam during the few seconds that the beam stayed in this machine. In the modified AA, the higher intensity and density made an efficient control of beam instability compulsory. Additional clearing by "beam shaking" and active damping of coherent instabilities were vital to reach the new design performance.

\subsection{Stochastic cooling and stacking}

The AA, and later also the AC, had large numbers of stochastic cooling systems: 7 in the original AA, 5 in the modified AA and 9 in the AC. As each had to be optimized for a specific task, their characteristics (pickup/kicker technology, bandwidth, gain, power, etc.) differed vastly. 
The precooling systems, acting on newly injected beams (less than $10^{8} \overline{\mathrm{p}}$ ), with time constants of a second, needed high-gain, high-power amplifiers. In the original AA, precooling acted only on momentum spread, using Thorndahl's notch-filter method [23]. Pickups and kickers consisted of short ferrite-frames around the beam, resembling beam transformers. Signals were coupled out or in, via "back-leg loops" on one side, the other leg being movable, acting as a shutter. A 2 m long tank contained 100 frames, and there were two kicker tanks and two pickup tanks. The bandwidth was 150-500 $\mathrm{MHz}$, and the amplifier rating $5 \mathrm{~kW}$.

In the AC, precooling was applied in all three phase planes. Pickups and kickers consisted of loop couplers, with electrodes left and right, or above and below the beam. The difference signal served for transverse cooling and the sum signal for momentum cooling. Two plate pairs connected in series formed a "superelectrode"; 24-48 of these (depending on their frequency band) were housed in $2 \mathrm{~m}$ long tanks. The electrodes moved in, to follow the shrinking beam size during cooling, thus maximizing pickup sensitivity and minimizing kicker power. The low-level components (pickups, terminations, preamplifiers) were cryogenically cooled to reduce noise. Three bands $(1-1.65,1.65-2.4$ and $2.4-3 \mathrm{GHz})$ were used, with three combined horizontal/momentum and three vertical/momentum systems, a total of 6 pickup and 6 kicker tanks. Amplifier ratings were $4.5 \mathrm{~kW}$ for the lower band and $2.6 \mathrm{~kW}$ for each of the higher bands, constituting a very-wide-band, high-power, low-noise, cryogenic HiFi-system, with feed-throughs and fast-moving electrodes in ultra-high vacuum.

The art of stacking by stochastic cooling and the solutions adopted for the AA are described in the literature [24, 25]. Stacking is done in momentum space: The pickups are located in regions of large dispersion, where particles are radially separated according to their momentum. Partial-aperture cooling systems acted over different aperture regions. The overall gain was profiled to decrease roughly exponentially from the low-density stack-tail (high gain) to the high-density core, by a factor comparable to the density ratio $\left(\approx 10^{5}\right)$. Loop couplers were used for the tail and Faltin-type slotted TEM lines [26] for the core. In the original AA, bands of $250-500 \mathrm{MHz}$ and $1-2 \mathrm{GHz}$ performed tail and core cooling, respectively.

When later precooling was done in the AC, simplifications became possible in the AA. In particular, shutters on the injection kicker and on the cooling devices at the injection orbit were no longer needed. Transverse cooling was less demanding, as beams of already low emittance arrived from the AC. On the other hand the higher intensity and density put greater demands on stack cooling. The performance was improved by further momentum precooling on the AA injection orbit and by a powerful transverse stack-core cooling system using partly the difference signal from the momentum systems. All cooling systems were replaced by higher frequency ones. More details can be found in the parameter lists [21, 22].

\subsection{Accumulator performance}

During its initial years of operation the AA performance progressed steadily. Routine operation was attained in 1982. For AC and AA together, this was the case in 1988. Table 2 reflects the performance in 1984 [21] and in 1994 [22]. Most impressive is the increase of phase space density (8-9 orders of magnitude) that was consistently obtained during cooling and accumulation. 
Table 2 - Operational performance of AA (in 1984) and AA + AC ( in 1994)

\begin{tabular}{|c|c|c|}
\hline Characteristic & $\begin{array}{l}\text { Original AA } \\
\text { (with horn) }\end{array}$ & $\begin{array}{c}\mathrm{AC}+\mathrm{AA} \\
(\text { with } 20 \mathrm{~mm} \text { lithium lens) }\end{array}$ \\
\hline $26 \mathrm{GeV} / \mathrm{c}$ protons per pulse on target $N_{\mathrm{p}}$ & $1.2 \times 10^{13}$ & $1.4 \times 10^{13}$ \\
\hline Antiprotons per pulse injected $N_{\overline{\mathrm{p}} / \mathrm{inj}}$ & $6.7 \times 10^{6}$ & $7.3 \times 10^{7}$ \\
\hline Antiproton yield $N_{\overline{\mathrm{p}} / \mathrm{inj}} / N_{\overline{\mathrm{p}}}$ & $5.6 \times 10^{-7}$ & $5.2 \times 10^{-6}$ \\
\hline Antiprotons stacked per injection $N_{\overline{\mathrm{p}}}$ stacked/inj & $4.7 \times 10^{6}$ & $6.0 \times 10^{7}$ \\
\hline Best daily production $\widehat{N}_{\overline{\mathrm{p}}}$ /day & $1.7 \times 10^{11 b}$ & $1.1 \times 0^{12 \mathrm{c}}$ \\
\hline Largest stack attained $\hat{N}_{\overline{\mathrm{p}}}$ & $2.8 \times 10^{11 \mathrm{~d}}$ & $1.3 \times 10^{12} \mathrm{e}$ \\
\hline Corresponding stacking factor ${ }^{\mathrm{f}} \widehat{N}_{\overline{\mathrm{p}}} / N_{\overline{\mathrm{p}} \text { stacked/inj }}$ & $6.0 \times 10^{4}$ & $2.2 \times 10^{4}$ \\
\hline $\begin{array}{l}\text { Increase of phase-space density }{ }^{\mathrm{g}} \\
\left(\bar{N}_{\overline{\mathrm{p}}} /\left(E_{\mathrm{h}} E_{\mathrm{v}} \Delta p\right)_{\text {stack }} /\left[N_{\overline{\mathrm{p}} \text { stacked } / \text { inj }} /\left(\mathrm{E}_{\mathrm{h}} E_{\mathrm{v}} \Delta p\right)_{\text {inj }}\right]\right.\end{array}$ & $5.4 \times 10^{8}$ & $4.3 \times 10^{9}$ \\
\hline
\end{tabular}

a With a $34 \mathrm{~mm}$ lithium lens the yield was $\sim 25 \%$ higher, with a horn $\sim 15 \%$ lower.

b Calculated from the above yield, assuming beam on target every $2.4 \mathrm{~s}$ (every PS cycle).

c Calculated assuming beam on target every $4.8 \mathrm{~s}$.

d November 1983.

e August 1989.

f Stacking factor $=$ number of pulses stacked.

$\mathrm{g}$ Taking the injected beam emittances equal to the acceptances (Table 1) and the stack core emittances as $E_{\mathrm{h}}=$ $E_{\mathrm{v}}=2 \pi \mathrm{mm} \operatorname{mrad}, \Delta p / p= \pm 1.5 \times 10^{-3}$.

\section{The SPS collider}

\subsection{Modifications to the SPS}

The SPS had barely seen its first beam in 1976, when the proposal came to convert it into a collider. As soon as the project was decided, in June 1978, work on the modifications [21] took a flying start:

- Most obvious was a new transfer line, TT70 (Fig. 2), to take the antiprotons from the PS to the SPS, and a new injection system there, for counter-clockwise injection.

- The SPS had been built for an injection momentum of $14 \mathrm{GeV} / \mathrm{c}$. The proton transfer line, TT10, and the injection system had to be upgraded to $26 \mathrm{GeV} / \mathrm{c}$.

- A drastic improvement of the vacuum was needed. On its design pressure, $2 \times 10^{-7}$ Torr for acceleration times counted in seconds, 2 orders of magnitude had to be gained for storage times counted in days.

- The machine lattice had to be modified to include "low-beta insertions", squeezing proton and antiproton beams to small sizes at the interaction points, for higher luminosity.

- The accelerating RF with its travelling-wave structures had to undergo modifications for simultaneous acceleration of protons and antiprotons. Precise synchronization between 
proton and antiproton bunches had to be implemented, for collisions to occur at the centre of the detectors.

- Beam diagnostics had to be adapted to very low intensities, and new devices added, such as directional couplers for independent observation of protons and antiprotons.

- The machine was affected by the digging of the underground areas for the experiments, the largest being those for UA1 and UA2, and the installation of the detectors with their huge magnets.

The SPS started up as a collider in 1981 at $273 \mathrm{GeV}$ (c.m. energy of $546 \mathrm{GeV}$ ), for the same r.m.s. magnet power as the $400 \mathrm{GeV}$ accelerator cycle. In 1984, increased water cooling allowed c.m. energy to be pushed to $630 \mathrm{GeV}$. For a short period of operation, a pulsed mode, cycling the energy between 100 and $450 \mathrm{GeV}$, extended the reach to c.m. energy of $900 \mathrm{GeV}$. Usually, there was one "fill" per day, and the beams were dumped when luminosity had become too low for the experiments to profitably take data.

Further modifications to the SPS Collider were made for the advent of the AC in 1987 and the tenfold increase in antiproton production.

- To reduce the limiting beam-beam effect, electrostatic deflectors separated beams everywhere except at the collision points inside the detectors.

- A new RF system at $100 \mathrm{MHz}$, half the accelerating frequency of $200 \mathrm{MHz}$, increased the longitudinal acceptance at injection. The more intense bunches (up to $10^{11} \overline{\mathrm{p}} / \mathrm{bunch}$ ) had a larger longitudinal emittance. Making them longer, the momentum spread was kept the same, to stay within the SPS momentum acceptance.

- Chromaticity correction was upgraded.

- In 1990, when UA2 took its last data, a "super-squeezed" low-beta scheme boosted luminosity by a further factor of 2 .

\subsection{Collider limitations and performance}

The figure of merit for a collider is the luminosity, $L$, the event rate per unit of cross-section. For head-on collisions of bunched beams $[\underline{11}, \underline{27}]$

$$
L=\frac{2 M N_{\mathrm{p}} N_{\overline{\mathrm{p}}} f \beta \gamma}{\pi\left[\beta_{\mathrm{h}} \beta_{\mathrm{v}}\left(\varepsilon_{\mathrm{h}_{\mathrm{p}}}^{*}+\varepsilon_{\mathrm{h}_{\overline{\mathrm{p}}}}^{*}\right)\left(\varepsilon_{\mathrm{v}_{\mathrm{p}}}^{*}+\varepsilon_{\mathrm{v}_{\overline{\mathrm{p}}}}^{*}\right)\right]^{1 / 2}}
$$

(usually given in $\mathrm{cm}^{-2} \mathrm{~s}^{-1}$ ). Here $M$ is the number of bunches per beam, $N_{\mathrm{p}}$ and $N_{\overline{\mathrm{p}}}$ the number of particles per bunch, $f$ the revolution frequency, $\beta_{\mathrm{h}, \mathrm{v}}$ the beta-function values at the collision point. The normalized emittances are defined as $\varepsilon_{\mathrm{h}, \mathrm{v}}^{*}=4 \beta \gamma \sigma_{\mathrm{h}, \mathrm{v}}^{2} / \beta_{\mathrm{h}, \mathrm{v}}$ with $\sigma$ the r.m.s. size of the beam. $L$ may differ between the intersection points, depending on the local beta values. A measure of the productivity is the integrated luminosity, $L_{\text {int }}$, which determines the number of events in a given time interval. It depends on the initial luminosity $L_{0}$, at the beginning of a fill, and on the luminosity lifetime $T_{L}$. For an exponential luminosity decay over an interval $T$

$$
L_{\text {int }}=L_{0} T_{L}\left[1-\exp \left(-T / T_{L}\right)\right] .
$$


As a unit one uses inverse micro-barn and inverse nano-barn $\left(1 \mathrm{nb}^{-1}=10^{33} \mathrm{~cm}^{-2}\right)$. The initial luminosity is determined both by the capabilities of the injector chain and by the SPS itself. The luminosity decay rate is proper to the SPS.

Limitations from the injector chain are mostly antiproton production rate and longitudinal and transverse emittances. These quantities depend on many parameters and an analysis is given in [11]. The antiproton production rate was raised significantly from 1987 onwards by the addition of the AC. The limitations within the SPS Collider itself were several [27]:

- The proton intensity was limited at injection by the "microwave instability". Above a threshold at about $9 \times 10^{10}$ protons per bunch, it caused a fast blow-up of the longitudinal emittance.

- Intra-beam scattering in the dense antiproton bunches caused growth of transverse and longitudinal emittances. It was the most determining effect for the luminosity decay rate, much more than Coulomb scattering on the residual gas, which, with the very good vacuum (eventually $2 \times 10^{-10}$ Torr), contributed little.

- Beam-beam interaction was a severe constraint. The highly non-linear field created by the intense proton bunches was felt by the antiprotons at the points of encounter and drove them towards resonances. Blow-up of their transverse emittances, even losses, was the consequence. The decay rate of antiproton density was thus highly sensitive to the working point. At a later stage, the electrostatic separators avoided unwanted encounters between protons and antiprotons, thereby reducing the beam-beam effect and allowing an increase from 3 bunches per beam to 6 . In the design phase, the beam-beam interaction had been a source of considerable worry. The SPS was the first to collide bunched hadron beams and the expectation that the beam-beam effect, much larger than at the ISR, would permit acceptable luminosity, had been one of the most daring assumptions in the project.

Operation of the Collider began in 1982 and performance increased significantly from year to year. A major step came in 1987/88, with the higher accumulation rate due to the AC and by further improvements to the SPS, mentioned above. Table 3 shows the performance in the years of operation with the big experiments (UA1 until 1989, UA2 until 1990):

Table 3 - SPS Collider performance and operational data

\begin{tabular}{|c|c|c|c|c|c|c|c|c|c|}
\hline \multirow[t]{2}{*}{ Characteristic } & \multicolumn{9}{|l|}{ Year } \\
\hline & 82 & 83 & 84 & 85 & 86 & 87 & 88 & 89 & 90 \\
\hline Beam energy $(\mathrm{GeV})$ & 273 & 273 & 315 & 315 & & 315 & 315 & 315 & 315 \\
\hline Beta functions $\beta_{\mathrm{h}}(\mathrm{m})$ & 1.5 & 1.3 & 1.0 & 1.0 & & 1.0 & 1.0 & 1.0 & 0.6 \\
\hline at collision point $\beta_{\mathrm{v}}(\mathrm{m})$ & 0.75 & 0.65 & 0.5 & 0.5 & & 0.5 & 0.5 & 0.5 & 0.15 \\
\hline \multirow[t]{2}{*}{ Number of bunches } & $3+3$ & $3+3$ & $3+3$ & $3+3$ & & $3+3$ & $6+6$ & $6+6$ & $6+6$ \\
\hline & & & & & & $6+6$ & & & \\
\hline Protons/bunch $\left(10^{10}\right)$ & 9.5 & 14 & 16 & 16 & & & 12 & 12 & 12 \\
\hline Antiprotons/bunch $\left(10^{10}\right)$ & 1.2 & 1.5 & 2 & 2 & & & 4 & 6 & 7 \\
\hline Average initial $L\left(10^{30} \mathrm{~cm}^{-2} \mathrm{~s}^{-1}\right)$ & 0.05 & 0.17 & 0.36 & 0.39 & & 0.35 & 1.3 & 1.8 & 3.1 \\
\hline$L_{\text {int }}$, average/coast $\left(\mathrm{nb}^{-1}\right)$ & 0.5 & 2.1 & 5.3 & 8.2 & & 2.8 & 31.5 & 40 & 70 \\
\hline$L_{\text {int }} /$ year $\left(\mathrm{nb}^{-1}\right)$ & 28 & 153 & 395 & 655 & 0 & 94 & 3608 & 4759 & 7241 \\
\hline Number of coasts/year & 56 & 72 & 77 & 80 & 0 & 33 & 107 & 119 & 104 \\
\hline Average duration of coast $(\mathrm{h})$ & 13 & 12 & 15 & 17 & & & 11 & 12 & 10 \\
\hline
\end{tabular}

Sources of data: $[11,27]$ and CERN Annual Reports 1982-1990. Notes: 1982: first year of routine operation; 1986: UA1 repair and beginning of AC installation; 1987: AC completion, only 7 weeks of "trial operation" at the end of the year; 1990: last year of full operation; 1991: only runs for UA4, UA6 of limited duration 
Representative for the amount of data taken by the experiments is the integrated luminosity. Figure 8 shows its evolution over the lifespan of the SPS Collider.

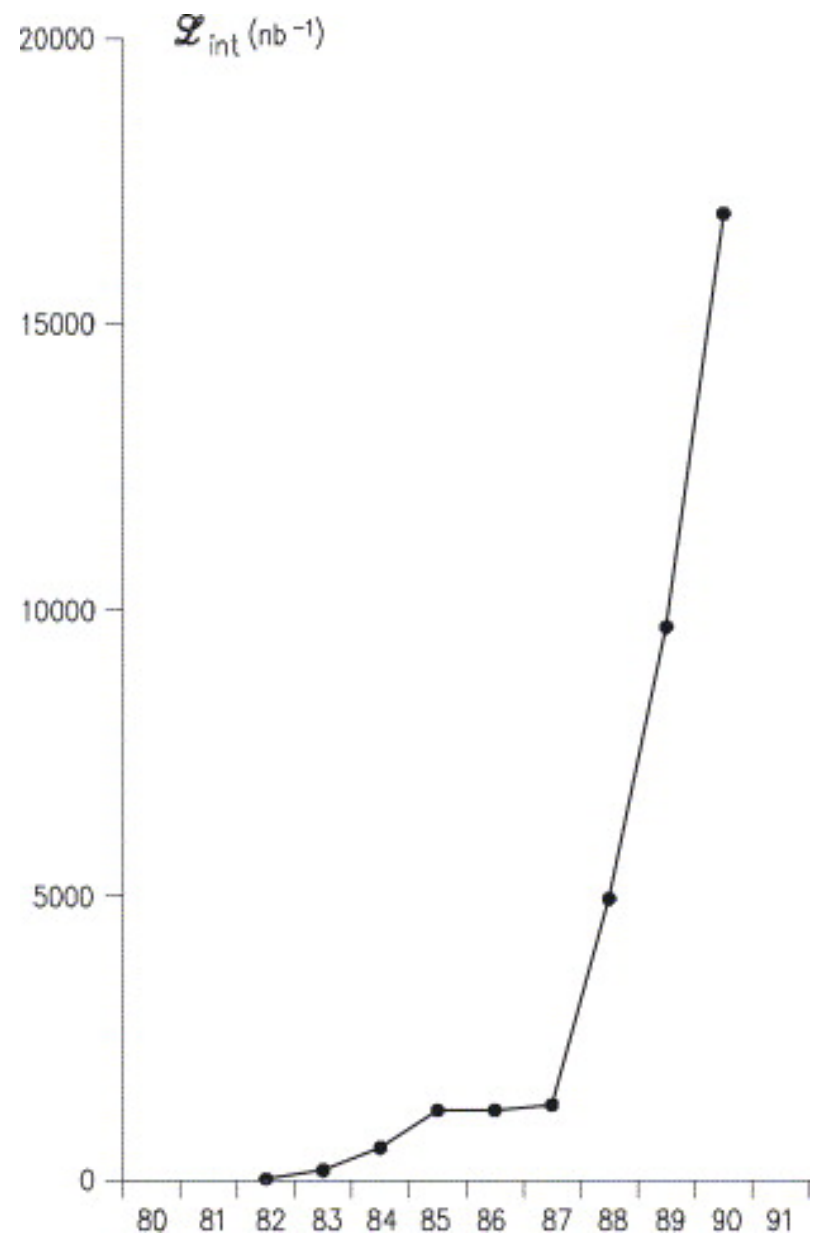

Fig. 8 - Integrated luminosity of the SPS Collider, from 1982 (first year of routine operation) to 1990 (last full operation). 1980 was the year of AA running-in, 1981 of Collider and detector tests. The luminosity integrated over 1982 and 1983 appears tiny, but sufficed to detect the $W$ and $Z$ and bring the Nobel prize 1984 to CERN. The break in 1986 was due to the repair of UA1 and the beginning of AC installation. AC running-in was completed in 1987, with only a short Collider run at the end of the year. From 1988 onwards, the effect of the AC and the improvements made to the SPS came to bear.

\section{Conclusions}

Looking back to the beginnings of CERN's Antiproton Collider Project, 28 years ago, one can only be amazed at the courage with which the project was attempted and the speed at which it was carried out. The prime driving force was the impatience to finally prove the existence of the intermediate bosons.

Apart from this main goal, the project opened the rich field of low-energy antiproton physics, which prospers still today [13], 15 years after the shut-down of the Collider experiments. And there is no doubt that the success of the antiproton programme, as another demonstration of CERN's accelerator competence, has greatly furthered the way towards the adventure of the Large Hadron Collider, the LHC.

There is another aspect that should be particularly pointed out: from the beginning, throughout the definition of the project, the construction of the accelerator and detector 
facilities, and the operation and data taking, there was an intense and most fruitful collaboration between the accelerator experts and the experimenters, and across all the Divisions involved. This breaking of the barriers was a source of motivation, which, no less than the scientific and technical effort and ingenuity, was essential for reaching success.

\section{References}

[1] K. Johnsen, Opening remarks, in: P. Bryant, S. Newman (Eds.), Proceedings of the CERN Acc. School, 1983, Antiprotons for Colliding Beam Facilities, CERN 84-15, 1984, p. 1.

[2] G.I. Budker, Status report of the work on storage rings at Novosibirsk, in: H. Zyngier, E. Cremieu-Alcan (Eds.), Proceedings of the Symposium International sur les Anneaux de Collision, Saclay 1966 (Presse Universitaire de France, Paris, 1966), p.II-1-1.

[3] S. van der Meer, Stochastic damping of betatron oscillations in the ISR, CERN Int. Report ISR-PO/72-31, 1972.

[4] G.I. Budker et al., Experimental study of electron cooling, IEEE Trans. Nucl. Sci. NS22 (1975), p. 2093.

[5] P. Bramham et al., Stochastic cooling of a stored beam, Nucl. Instrum. Methods $\mathbf{1 2 5}$ (1975), p. 201.

[6] C. Rubbia, P. McIntyre, D. Cline, Producing massive intermediate vector bosons with existing accelerators, in: Proceedings of the International Neutrino Conference, Aachen 1976, Vieweg Verlag, Braunschweig, 1977, p. 683.

[7] G. Carron et al., Stochastic cooling tests in ICE, Phys. Lett. 77B (1978), p. 353.

[8] P. Strolin, L .Thorndahl, D. Möhl, Stochastic cooling of antiprotons for ISR physics, CERN Int. Report EP 76-05, 1976.

[9] M. Bregman et al., Measurement of antiproton lifetime using the ICE storage ring, Phys. Lett. 78B (1978), p. 174.

[10] AA design study team, Design of a proton-antiproton colliding beam facility, CERN Int. Report PS/AA 78-3, 1978.

[11] L. Evans, E. Jones, H. Koziol, The CERN p $\bar{p}$ Collider, in: G. Altarelli, L. di Lella (Eds.), Proton-Antiproton Collider Physics, World Scientific Publishing Co., Singapore, 1989.

[12] E.J.N. Wilson (Ed.), Design study of an Antiproton Collector for the Antiproton Accumulator, CERN 83-10, 1983.

[13] H. Koziol, D. Möhl, The CERN low-energy antiproton programme: the synchrotrons; to be published in Phys. Rep.; preprint CERN-AB-2004-098 ADM, 2004

[14] J. Allaby, Antiproton production, in: P. Bryant, S. Newman (Eds.), Proceedings of the CERN Acc. School, 1983, Antiprotons for Colliding Beam Facilities, CERN 84-15, 1984, p.63.

[15] C. Hojvat and A. van Ginneken, Calculation of antiproton yields for the Fermilab antiproton source, Nucl. Instrum. Methods 206 (1983), p. 67.

[16] M.D. Church and J.P. Marriner, The antiproton sources: design and operation, Ann. Rev. Nucl. Part. Sci. 43 (1995), p. 253. 
[17] S. van der Meer, Improved collection of secondaries from a long, narrow target by a horn doublet, CERN Int. Report PS/AA 80-12, 1980.

[18] D. Boimond, et al., Consolidation of the $400 \mathrm{kA}$ magnetic horn for AAC antiproton production, CERN Int. Report PS 94-02-AR, 1994.

[19] R. Bellone, et al., Beam tests of a $36 \mathrm{~mm}$ lithium lens, in: Proceedings of the Second European Particle Accelerator Conference EPAC '90, Nice, France, 1990, p.1303; R. Bellone, et al., Performance and operational experience with CERN-lithium lenses, in: Proceedings of the First European Particle Accelerator Conference EPAC '88, Rome, 1988, p. 1401

[20] R. Kowalewicz, et al., Beam tests with the CERN plasma lens, in: Proceedings of the Third European Particle Accelerator Conference, EPAC '92, Berlin, 1992, p. 1539.

[21] H. Koziol, Antiproton Accumulator (AA) Parameter List, 9th Edition, CERN Int. Note PS/AA/Note 80-2, 1980; 10th Edition, CERN Int. Note PS/AA/Note 84-2, 1984.

[22] S. Maury, H. Koziol, Parameter list for the Antiproton Accumulator Complex (AAC), CERN Int. Report /PS 95-15 (AR/BD), 1995.

[23] G. Carron, L. Thorndahl, Stochastic cooling of momentum spread with filter methods, CERN Int. Report /ISR-RF/ 78-12, 1978.

[24] S. van der Meer, Stochastic stacking in the Antiproton Accumulator, CERN Int. Report PS/AA 78-22, 1978.

[25] F. Caspers and D. Möhl, Stacking with stochastic cooling, in: Proceedings of the International Workshop on Beam Cooling and Related Topics, Nucl. Instrum. Methods A 532 (2004), p. 321.

[26] L. Faltin, Slot-type pick-up and kicker for stochastic beam cooling, Nucl. Instrum. Methods 148 (1978), p. 449.

[27] L. Evans, The SPS collider: status and outlook, in: Proceedings of the 1987 Particle Accelerator Conference, Washington D.C., 1987, p. 2428. 